\title{
Bronquiolite obliterante em paciente pediátrico associada com exposição à cocaína e outras drogas de abuso durante o período gestacional
}

\author{
Bronchiolitis obliterans in pediatric patient associated with exposure to cocaine and other \\ abuse drugs during the gestational period \\ Bronquiolitis obliterante en paciente pediátrico asociada con la exposición a cocaína y otras \\ drogas de abuso durante el período gestacional
}

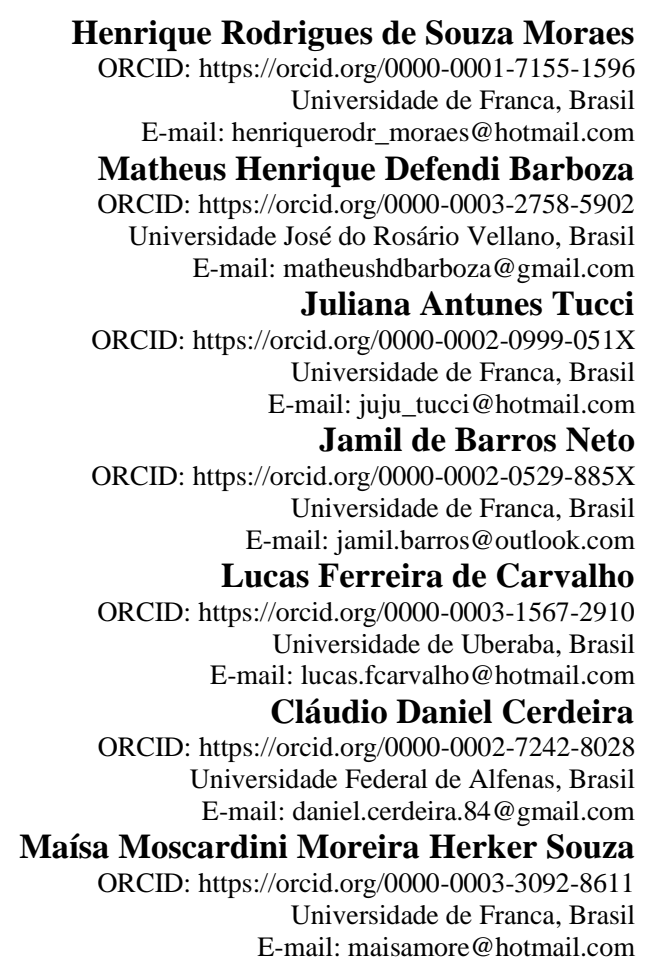

\begin{abstract}
Resumo
São ainda escassas as informações na literatura acerca da associação entre exposição da gestante a drogas de abuso, incluindo a cocaína, e o desenvolvimento de bronquiolite obliterante (BO) no neonato. Neste relato de caso abordamos o acompanhamento ao longo dos anos de um paciente pediátrico do sexo masculino que deu entrada como lactente sibilante e, que, após avaliações e observância de critérios clínicos e por imagem sugestivos, foi diagnosticado com BO. Portanto, os achados deste relato fomentam a literatura e lançam uma base acerca de uma relação causal entre exposição da gestante a drogas de abuso e o desenvolvimento de BO no recém-nascido, bem como sobre o perfil epidemiológico, diagnóstico clínico e por imagem e terapêutica (medicamentos e oxigenoterapia) no curso da doença em paciente pediátrico e, suas comorbidades, direcionando também futuras estratégias para o manejo dos pacientes com esta doença.

Palavras-chave: Bronquiolite obliterante; Cocaína; Gestação; Pediatria.
\end{abstract}

\begin{abstract}
There is still little information in the literature about the association between exposure of pregnant women to drugs of abuse, including cocaine, and the development of bronchiolitis obliterans (BO) in the neonate. In this case report, we covered the follow-up over the years of a male pediatric patient who was admitted as a wheezing infant and who, after evaluations and observance of suggestive clinical and imaging criteria, was diagnosed with BO. Therefore, the findings of this report foster the literature and shed light on a causal relationship between exposure of pregnant women to drugs of abuse and the development of $\mathrm{BO}$ in the
\end{abstract}


newborn, as well as on the epidemiological profile, clinical and imaging diagnosis and therapy (medications and oxygen therapy) in the course of the disease in pediatric patients and their comorbidities, also directing future strategies for the management of patients with BO.

Keywords: Bronchiolitis obliterans; Cocaine; Gestational age; Pediatrics.

\section{Resumen}

Todavía hay poca información en la literatura sobre la asociación entre la exposición de mujeres embarazadas a drogas de abuso, incluida la cocaína, y el desarrollo de bronquiolitis obliterante (BO) en el recién nacido. En este reporte de caso, cubrimos el seguimiento a lo largo de los años de un paciente pediátrico masculino que ingresó como un lactante con sibilancias y que, luego de evaluaciones y observación de criterios clínicos y de imagen sugestivos, fue diagnosticado de BO. Por lo tanto, los hallazgos de este informe fomentan la literatura y proporcionan una base para una relación causal entre la exposición de las mujeres embarazadas a drogas de abuso y el desarrollo de $\mathrm{BO}$ en el recién nacido, así como sobre el perfil epidemiológico, diagnóstico y tratamiento clínico e imagenológico (medicamentos y oxigenoterapia) en el curso de la enfermedad en pacientes pediátricos y sus comorbilidades, orientando también las estrategias futuras para el manejo de los pacientes con esta enfermedad.

Palabras clave: Bronquiolitis obliterante; Cocaína; Gestación; Pediatría.

\section{Introdução}

A Bronquiolite Obliterante (BO) é uma condição rara e caracterizada, estruturalmente, conforme seu título indica: patogenia com lesão inflamatória não específica que afeta as pequenas vias aéreas inferiores, com progressão ao interstício axial e septal dos pulmões e presença marcante de fibrose das paredes bronquiolares, o que leva a obstrução do lúmen bronquiolar e, funcionalmente, a uma limitação da capacidade respiratória. Foi primeiramente descrita por Lange em 1901, em dois pacientes que se apresentavam com doença pulmonar idiopática. Na infância a BO cursa com remodelamento (fibrose) das pequenas vias aéreas após uma agressão que resulte em processo inflamatório agudo, levando à obstrução do fluxo aéreo (Garipo \& Capelozzi, 2006; Lobo et al., 2007; Silva \& Müller, 2008; Lino et al., 2013).

A BO na faixa etária pediátrica possui incidência desconhecida e acomete principalmente lactentes do sexo masculino (Santos et al., 2004; Aguerre et al., 2010; Lino et al., 2013). A literatura atual carece de dados epidemiológicos fidedignos, mas conforme alguns estudos, a BO pode apresentar uma considerável prevalência no Brasil, pois é amplamente relacionada a doença pulmonar obstrutiva crônica (DPOC) da infância, aumentando a demanda de atendimentos ambulatoriais e hospitalares (Lino et al., 2013). Além disso, a literatura cita a América do Sul como sendo uma região de considerável prevalência de BO (Lobo et al., 2007).

Quanto as possíveis etiologias, quando não idiopática, a BO pode ter como causa a inalação de substâncias tóxicas; síndromes aspirativas; imunodeficiências, síndrome de Sjögren e artrite reumatóide; alterações do metabolismo de colágeno e de tecido conjuntivo; causas do pós-transplante e pós-operatório e; pode também ser induzida por drogas e medicamentos (Terra Filho et al., 2004; Garipo \& Capelozzi, 2006; Lin et al., 2018; Callahan et al., 2019). Contudo, durante a infância, a BO pós-infecciosa nas vias aéreas inferiores se destaca, ocorrendo também em crianças imunocomprometidas, sendo causada principalmente pelos adenovírus 3, 7 e 21, sendo citada na pós infecção pelo vírus sincicial respiratório, além de algumas bactérias, como Mycoplasma pneumoniae, Streptococcus tipo B, Legionella pneumophila e Bordetella pertussis (Teper et al., 2002; Lobo et al., 2007; Colom \& Teper, 2009a; Colom \& Teper, 2009b; Fischer et al., 2010; Champs et al., 2011; Lino et al., 2013). Além disso, pneumonias causadas por microrganismos oportunistas destacam-se como comorbidades em pacientes com BO, além de um diferenciado microbioma com a presença de Streptococcus pneumoniae, Haemophilus influenzae e Moraxella catharralis (Lino et al., 2013). 
Dados quanto ao uso de cocaína pela gestante e a relação com a BO em neonatos são ainda escassos na literatura atual, mas sabe-se que o abuso de drogas está relacionado à agressões contra a parede do trato respiratório, levando a um processo inflamatório secundário (Terra Filho et al., 2004; Garipo \& Capelozzi, 2006). Neste relato apresentamos o caso de um paciente pediátrico diagnosticado com BO, discutindo a relação entre exposição à cocaína durante a gestação e o desenvolvimento de BO no recém-nascido, acrescentando dados quanto ao perfil clínico e diagnóstico por imagem, epidemiológico e de tratamento da $\mathrm{BO}$ em tal faixa etária.

\section{Relato do Caso}

Este relato de caso aborda o acompanhamento de um paciente pediátrico, AHMS, sexo masculino, diagnosticado com BO e, fazendo uso de cateter de oxigênio, atualmente com 4 anos, que deu entrada na Santa Casa de um município paulista, como lactente sibilante, apresentando inicialmente queixas de tosse copiosa não produtiva, dispneia a pequenos esforços e roncos durante o sono, no mais, sono regular e sem alterações. Acompanhamento iniciou-se em 2016 e, estende-se até o momento. Na história médica (2018) relatou-se: crise de bronquiolite e pneumonia, sendo que em agosto do mesmo ano, o paciente foi internado com uma pneumonia lobar direita, com posterior resolução.

Histórico gestacional: durante a gestação, a mãe fazia uso de cigarros, maconha, cocaína e álcool, nega traumas físicos e realizava pré-natal. Em 05/11/2016, paciente nasceu de parto normal, com uma gestação em torno de 37 semanas e três dias, Swab + com uso de ampicilina antes do parto, APGAR 8 e 9, choro ao nascimento sem hipotonia, peso ao nascer de $2915 \mathrm{~g}$ e altura de $49 \mathrm{~cm}$. Fez fototerapia por 5 dias. No mais, parto sem intercorrências.

Em junho de 2018 foi verificado o histórico do paciente: carteira de vacinação em dia. Nega alergias e outras afecções do sistema respiratório. Alimentação com leite de vaca. IMC de 25. Paciente sem histórico de uso de substâncias sistêmicas ou inaladas, exceto medicamentos, ou de viagem recente. Desenvolvimento neuropsicomotor (DNPM) adequado para a faixa etária. Relata sudorese excessiva desde início do uso das medicações abaixo indicadas. Responsável legal foi orientado para (re)adequação do ambiente quanto a presença de possíveis alérgenos.

Em 25/09/2018, a gasometria arterial foi avaliada: $\mathrm{pH}=7,39, \mathrm{pCO}_{2}=40,5, \mathrm{pO}_{2}=63,4, \mathrm{BE}:-1,1 / \mathrm{HCO}_{3}=23,8$, $\mathrm{FiO}_{2}=0,210 \%$. Ao exame físico o paciente apresentava-se com "bom estado geral" B.E.G. (ativo e reativo), afebril, corado, hidratado, anictérico, Sat $\mathrm{O}_{2}=99 \%$, e sem cansaço e cianose. Fácies cushingoide, ACV: 2BRNF 2T S/S, AR: MV+, simétricos bilateralmente, raros sibilos e ausência de estertores e crepitações. Abdômen (ABD): flácido, indolor a palpação, sem visceromegalias, com fígado e baço não palpáveis. Medicações em uso pelo paciente: Prednisolona 3 $\mathrm{mg} / \mathrm{mL}$ ( $5 \mathrm{~mL}$ ), Salbutamol $100 \mathrm{mcg}$ ( 4 puffs de $6 / 6 \mathrm{~h}$, com auxílio de espaçador e máscara), Clenil $50 \mathrm{mcg}$ ( 2 puffs de 12/12 h, com auxílio de espaçador e máscara), e Azitromicina $200 \mathrm{mg} / 5 \mathrm{~mL}$. Posteriormente, ao exame físico, paciente manteve-se com os mesmos parâmetros, com exceção da presença de secreção hialina no nariz e ausência de hipertrofia de cornetos, presença de estertores finos na ausculta e a $\mathrm{Sat}_{2}$ caiu para $92 \%$, com frequência cardíaca de 49 , sendo que a oxigenoterapia foi mantida e acrescentado naquele momento no arsenal terapêutico do paciente a Ranitidina e Domperidona. Solicitou-se retorno em 3 meses. Neste intervalo, ocorreram episódios de broncoespasmo.

Considerando que o paciente inicialmente deu entrada como lactente sibilante, na sequência, os achados clínicos e radiográficos direcionaram o diagnóstico, com destaque para o fator de risco para a $\mathrm{BO}$, a história de uso de drogas de abuso pela mãe durante a gestação. Além disso, na tomografia computadorizada (TC) (Figura 1), constatou-se áreas de aprisionamento aéreo, juntamente com áreas de opacidade apresentando padrão em vidro fosco + fibrose e atelectasia cilíndrica, em lobo superior esquerdo e segmento superio-medial de lobo direito. Um padrão de mosaico 
pode ser observado, com áreas de hiper e hipoatenuação nos pulmões, representando lesões nas pequenas vias aéreas. Com os achados característicos na TC, e os dados clínicos e histórico médico/familiar, confirmou-se o diagnóstico de BO.

Na evolução do caso, em 19/02/2019 relatou-se tosse seca, predominantemente noturna, acompanhada de rinorreia. Esalerg (2,5 mL/10 dias) foi receitado, com manutenção dos demais medicamentos. 19/03/2019: foi relatado

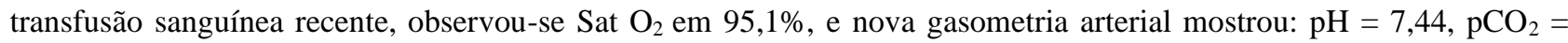
$37,2, \mathrm{pO}_{2}=70,8, \mathrm{BE}: 0,7 / \mathrm{HCO}_{3}=24,7, \mathrm{FiO}_{2}=0,210 \%$. Ao exame físico paciente apresentava-se novamente com B.E.G. ativo e reativo, afebril, corado, hidratado, anictérico, sem cansaço e cianose. 2BRNF 2T, ausência de sopros. AR: MV+, simétricos bilateralmente, presença de estertores bilateralmente e sibilos expiratórios. ABD: flácido, indolor a palpação, sem visceromegalias. Orofaringe: presença de cárie dentária em vários dentes, sem mais alterações. Exames laboratoriais: $\mathrm{TGO}=26$; $\mathrm{TGP}=16 ; \mathrm{TSH}=1,11 ;$ Ureia $=23$; Creatinina $=06$; Glicemia em jejum $=95$. Quanto a terapia, manteve-se os medicamentos e oxigenoterapia.

Figura 1 - Tomografia computadorizada (TC) de tórax do paciente pediátrico.

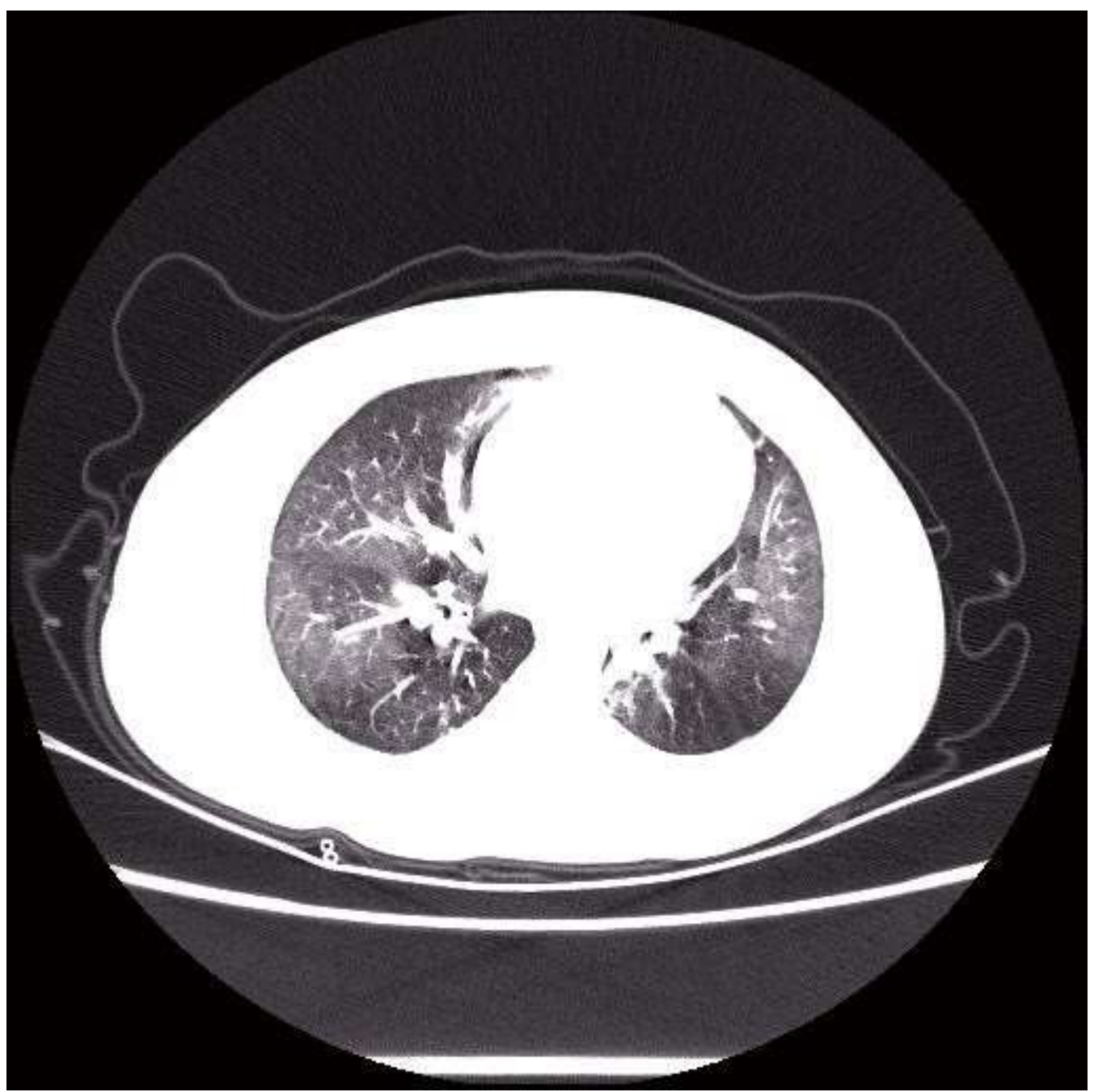

Fonte: Autores.

Em 04/2019: paciente foi diagnosticado com pré-Diabetes mellitus (DM), tendo sido orientado uma dieta restritiva de açucares e encaminhado ao nutricionista. Em novo exame físico, observou-se paciente com B.E.G. (ativo e 
reativo); afebril; corado; hidratado; anictérico; e sem cansaço e cianose. Fácies cushingoide. Oroscopia e otoscopia sem alterações. AR: MV+, presença de estertor bolhoso em base de pulmões. ABD: Globoso, rígido sem herniações, RHA+, sem alterações a palpação e percussão. Comorbidades: Genitália: fimose 1; criptorquidite. Lesões hiperemiadas transicionando para hipercrômicas em região perior, MMSS (região das mãos), MMII (região meleolar) e assadura em região geniturinária, sob uso de Clotrimazol pomada $(10 \mathrm{mg} / \mathrm{g})$.

Após aumento de dose da Prednisolona ( $3 \mathrm{mg} / \mathrm{mL}, 1 \mathrm{mg} / \mathrm{Kg} / \mathrm{dia}=6 \mathrm{~mL}$ ), em 25/06/2019, optou-se por ajustes com uma leve diminuição na dose do medicamento para $5 \mathrm{~mL}$ (3 mg/mL, 1x dia, 0,97 mg/Kg/dia). Em 17/09/2019, paciente deu nova entrada por pneumonia. No fim de 2019 foi realizado um esclarecimento ao responsável quanto a necessidade constante da oxigenoterapia em cateter nasal (domicílio e creche). Paciente foi também encaminhado ao cirurgião pediátrico, com retorno em 1 mês. Desde julho de 2019 sob fisioterapia.

\section{Discussão}

Neste relato de caso acompanhamos um paciente pediátrico com $\mathrm{BO}$, o qual foi exposto durante seu desenvolvimento gestacional à cocaína e outras drogas de abuso; relatando a história da doença, no decorrer de aproximadamente 4 anos. Na literatura, existem poucos dados fidedignos a respeito da evolução natural da BO na criança, bem como quanto a etiologia da doença sendo causada por exposição materna a drogas de abuso durante o período gestacional, destacando a importância deste relato. Normalmente, a BO está associada a outras etiologias, como exemplo, lactentes com idade inferior a um ano, que são admitidos em pronto atendimento, do tipo sazonal, são diagnosticados com BO induzida pela infecção pelo vírus respiratório sincicial, adenovírus, além de outros microrganismos e, cursam com o desenvolvimento de sequelas, como a DPOC (Chang et al., 1998; Garipo \& Capelozzi, 2006).

A patogenia da BO é bem definida, sendo desencadeada por agressões à parede do trato respiratório, levando a um processo inflamatório, precedendo a proliferação de tecido de granulação, o qual ocasiona um estreitamento do lúmen das vias respiratórias inferiores e pode gerar uma obstrução completa dos bronquíolos por fibrose submucosa (Garipo \& Capelozzi, 2006; Lino et al., 2013). Tem sido descrito que o uso da cocaína se relaciona com as alterações no epitélio respiratório e, a intensidade das consequências, depende das vias de administração, tempo de exposição e quantidade de uso (de Almeida et al., 2002). Ainda, esta droga quando em contato com o epitélio respiratório inferior pode causar vasoconstrição das artérias pulmonares, seguido de anóxia celular, além do possível efeito tóxico, direto no endotélio capilar alveolar e brônquico e, reação de hipersensibilidade, induzida por seus componentes. As complicações causadas pela cocaína podem ainda estar relacionadas a infecções oportunistas, por exemplo, por Staphylococcus aureus (Terra Filho et al., 2004). Não existem ainda evidências concretas quanto ao uso materno de drogas de abuso (incluindo a cocaína) e complicações respiratórias em recém-nascidos, embora existam especulações (Terra Filho et al., 2004). Portanto, aqui reportamos a associação entre exposição da gestante a cocaína e outras drogas de abuso durante a gestação e o desenvolvimento de BO no neonato.

No acompanhamento e diagnóstico de $\mathrm{BO}$ no paciente pediátrico, observou-se variação no perfil quanto aos parâmetros clínicos e, consequentemente, foram necessárias mudanças no protocolo terapêutico. O curso clínico da doença é variável, indo desde formas leves com evolução favorável até formas graves, com complicações fatais (Lino et al., 2013), com persistência em graus variáveis da disfunção respiratória (Lino et al., 2013), portanto, pacientes hipoxêmicos crônicos $\left(\mathrm{SpO}_{2}<94 \%\right)$ devem estar sob constante oxigenoterapia (Lino et al., 2013). Hipoxemia e dependência de oxigênio são comuns em pacientes com BO, podendo persistir nas formas mais graves por longos 
períodos (Lino et al., 2013), como observado no presente relato. Neste contexto, orientações e instruções foram também direcionadas ao responsável e familiares do paciente pediátrico acerca da importância da oxigenoterapia no curso do tratamento, uma vez que o paciente, segundo monitoramento, apresentava-se grande parte do tempo hipoxêmico.

Além da história familiar, a clínica e outros achados, como os provenientes do diagnóstico por imagem, foram essenciais para orientar o diagnóstico final de BO. É descrito que a doença, frequentemente, apresenta um quadro clássico de tosse, associada à sibilância, estertores e anormalidades radiológicas como espessamento peribrônquico, hiperinsuflação, bronquiectasias e atelectasia. A BO é caracterizada em achados radiográficos por inflamação e fibrose das vias aéreas com diâmetro $<2 \mathrm{~mm}$. Na infância, ela tem um caráter predominantemente constrictivo (Garipo \& Capelozzi, 2006; Silva \& Müller, 2008; Lino et al., 2013; Callahan et al., 2019). No diagnóstico de BO, possíveis diagnósticos diferenciais devem ser levados em conta, tais como o refluxo gastroesofágico, fibrose cística, tuberculose pulmonar e deficiência de alfa-1-antitripsina. O diagnóstico diferencial mais importante é a sibilância recorrente pósbronquiolite, diferenciada a partir de dados clínicos. Na BO existe uma sintomatologia persistente, estertores finos, assim como alterações radiológicas características, sendo a resposta ao uso de broncodilatadores durante a espirometria insatisfatória e o prognóstico desfavorável (Zhang \& Silva, 2000). A TC, utilizada no presente caso, é considerada um exame adequado para avaliar as lesões presentes na BO, sendo possível avaliar o espessamento brônquico, bronquiectasia, atelectasia e áreas mistas de hipo e hiperatenuação, referida como perfusão em mosaico ou padrão em mosaico de atenuação pulmonar, sendo este o sinal mais notável em patologias que cursam com lesões das vias aéreas inferiores e, compatíveis com os achados descritos no presente relato (Zhang \& Silva, 2000; Smith et al., 2010).

Quanto ao tratamento, como observado no presente estudo, o paciente pediátrico desenvolveu uma síndrome de Cushing (observada no exame físico como "fácies cushingoide"), em decorrência do uso crônico de doses imunossupressoras de corticoide (Prednisolona). Ainda como uma consequência de tal terapêutica, relatou-se o desenvolvimento de pré-DM pelo paciente e uma tendência à obesidade. O tratamento da BO ainda é pouco compreendido, por envolver vários sintomas com evolução clínica variada, sendo o uso de corticoide comum (Calabrese et al., 2016). Sobre a corticoterapia sistêmica, existem raros ensaios clínicos que avaliam os diversificados efeitos e possíveis danos desta terapêutica nos pacientes com BO e, por falta de evidência associada aos possíveis efeitos colaterais, o uso deve ser cauteloso. No presente relato, houve ajustes das doses de acordo com a evolução clínica do paciente. É também descrito um efeito significativo em pacientes pela diminuição do número de neutrófilos no lavado broncoalveolar e pela melhoria na função pulmonar (Zhang \& Silva, 2000). Devido a semelhança entre as características pulmonares verificadas por TC de alta resolução na BO e as encontradas em pacientes com a doença causada pelo novo coronavírus [SARS-CoV-2 (do inglês $\underline{\text { Severe }}$ Acute Respiratory $\underline{\text { Syndrome }}$ Coronavirus-2)],

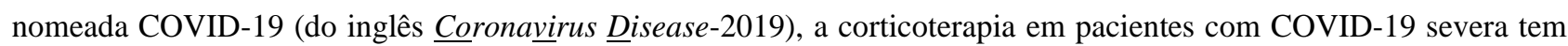
sido justificada embasada em tais achados por imagem (Zheng et al., 2020).

Além disso, a pneumonia microbiana causada por patógenos verdadeiros ou microrganismos oportunistas é comum em pacientes com BO, sendo também observado um microbioma peculiar nesses pacientes (Lino et al., 2013), justificando assim o uso da azitromicina no tratamento complementar, conforme observado no presente relato de caso, sendo que este antimicrobiano também mostrou-se, em prévios estudos, capaz de atenuar a obstrução do fluxo aéreo por diminuir o infiltrado de neutrófilos e a concentração de IL-8 (Yates et al., 2005; Verleden et al., 2006). Na BO pósinfecciosa ocorre um predomínio de neutrófilos e linfócitos T CD8+ e níveis elevados das interleucinas IL-6 e IL-8.

Como aqui observado, comorbidades não relacionadas podem ocorrer no paciente com $\mathrm{BO}$, como visto nas alterações em: genitália; fimose 1, assadura em região geniturinária e criptorquidite; e lesões hiperemiadas 
transicionando para hipercrômicas em região perior, mãos e meleolo. Comorbidades em pacientes com BO são também descritas na literatura (Kim \& Lee, 1996; Callahan et al., 2019), podendo também a própria doença predispor o paciente a comorbidades ou outras doenças induzir BO (Teper et al., 2002; Lin et al., 2018). A promoção de uma melhoria na qualidade de vida dos pacientes com BO deve ser estimulada, visto as complicações da doença bem como as possíveis comorbidades que eles enfrentam (Sarria et al., 2018).

\section{Considerações Finais}

Os achados deste relato fomentam a literatura acerca de uma relação causal entre exposição da gestante a cocaína e outras drogas de abuso e o desenvolvimento de BO no recém-nascido, bem como sobre o perfil epidemiológico, diagnóstico clínico e por imagem e terapêutica (medicamentos e oxigenoterapia) no curso da doença em paciente pediátrico e, suas comorbidades, direcionando também futuras estratégias para o manejo dos pacientes com BO.

\section{Agradecimentos}

Gostaríamos de agradecer ao paciente e familiares, bem como a todos que contribuíram com o presente relato.

\section{Aspectos éticos}

Os envolvidos diretos, paciente e familiares, foram esclarecidos sobre os propósitos do relato, oportunidade na qual um responsável assinou o TCLE. Este relato foi aprovado pelo comitê de ética em pesquisa do grupo Santa Casa de Franca (parecer \#4.829.884).

\section{Referências}

Aguerre, V., Castaños, C., Pena, H. G., et al. (2010). Postinfectious bronchiolitis obliterans in children: clinical and pulmonary function findings. Pediatr Pulmonol, 45, 1180-5.

Calabrese, C., Corcione, N., Rea, G., et al. (2016). Impact of long-term treatment with inhaled corticosteroids and bronchodilators on lung function in a patient with post-infectious bronchiolitis obliterans. J Bras Pneumol, 42(3), 228-31.

Callahan, S. J., Vranic, A., Flors L., et al. (2019). Sporadic Obliterative Bronchiolitis: Case Series and Systematic Review of the Literature. Mayo Clin Proc Inn Qual Out, 3(1), 86-93

Champs, N. S., Lasmar, L. M. L. B. F., Camargos, C. M., et al. (2011). Post-infectious bronchiolitis obliterans in children. Jornal de Pediatria, 87(3), 187-198.

Chang, A. B., Masel, J. P., \& Masters, B. (1998). Post-infectious bronchiolitis obliterans: clinical, radiological and pulmonary function sequelae. Pediatr Radiol, 28, 23-9.

Colom, A. J., \& Teper, A. M. a. (2009). Postinfectious bronchiolistis obliterans. Arch Argent Pediatr, 107, 160-7

Colom, A. J., Teper, A. M. b. (2009). Clinical prediction rule to diagnose post-infectious bronchiolitis obliterans in children. Pediatr Pulmonol, 44, 1065-9.

de Almeida, P., Guimarães, M. J. B., Costa, M. G., et al. (2002). Bronquiolite obliterante na forma nodular. J Pneumol, 28(6), 335-8.

Fischer, G. B., Sarria, E. E., Mattiello, R., et al. (2010). Post Infectious bronchiolitis obliterans in children. Paediatr Respir Rev, 11, $233-9$.

Garipo, A. L., \& Capelozzi, V. L. (2006). Estudo morfométrico da imunidade celular e remodelamento no eixo pré-acinar na indução do colágeno tipo $\mathrm{V}$ em um modelo de bronquiolite obliterante. $186 \mathrm{f}$. Tese (Doutorado) - Curso de Fisiopatologia Experimental, Faculdade de Medicina da Universidade de São Paulo, São Paulo, 2006.

Kim, M. J., \& Lee, K. Y. (1996). Bronchiolitis obliterans in children with Stevens-Johnson syndrome: follow-up with high resolutions CT. Pediatr Radiol, 26, 22-5.

Lin, E., Limper, A. H., \& Moua, T. (2018). Obliterative bronchiolitis associated with rheumatoid arthritis: analysis of a singlecenter case series. BMC Pulmonary Medicine, 18, 105. 
Research, Society and Development, v. 10, n. 9, e35210918021, 2021

(CC BY 4.0) | ISSN 2525-3409 | DOI: http://dx.doi.org/10.33448/rsd-v10i9.18021

Lino, C. A., Batista, A. K. M., Soares, M. A. D., et al. (2013). Bronquiolite obliterante: perfil clínico e radiológico de crianças acompanhadas em ambulatório de referência. Rev Paul Pediatr, 31(1), 10-16.

Lobo, A. L., Guardiano, M., Nunes, T., et al. (2007). Bronquiolite obliterante pós-infecciosa na criança. Revista Portuguesa de Pneumologia, 13(4), 495-509.

Santos, R. V., Rosário, N. A., \& Ried, C. A. (2004). Postinfectious bronchiolitis obliterans: clinical aspects and complementary tests of 48 children. $J$ Bras Pneumol, 30, 20-5.

Sarria, E. E., Mundstock, E., Machado, D. G., et al. (2018). Health-related quality of life in patients with bronchiolitis obliterans. J Pediatr, 94(4), 374-379.

Silva, C. I. S., \& Müller, N. L. (2008). Obliterative Bronchiolitis. Springer, 293-323. In: Contemporary Medical Imaging: CT of the Airways, Edited by: P. M. Boiselle and D. A. Lynch ${ }^{\odot}$, Humana Press, Totowa, NJ (2008).

Smith, K. J., Dishop, M. K., Fan, L. L., et al. (2010). Diagnosis of bronchiolitis obliterans with computed tomography in children. Pediatr Allergy Immunol Pulmonol, 23, 253-9.

Teper, A., Fischer, G. B., \& Jones, M. H. (2002). Seqüelas respiratórias de doenças virais: do diagnóstico ao tratamento. Jornal de Pediatria, 78, 187194.

Terra Filho, M., Yen, C. C., Santos, U. P., et al. (2004). Alterações pulmonares em usuários de cocaína. Sao Paulo Medical Journal, 122(1), $26-31$.

Verleden, G. M., Vanaudenaerde, B. M., Dupont, L. J., et al. (2006). Azithromycin reduces airway neutrophilia and interleukin-8 in patients with bronchiolitis obliterans syndrome. Am J Respir Crit Care Med, 174, 566-70.

Zhang, L., Silva, F. A. (2000). Bronquiolite obliterante em crianças: bronchiolitis obliterans in children. Jornal de Pediatria, 76(3), 185-192.

Zheng, Z., Yao, Z., Wu, K., et al. (2020). The diagnosis of pandemic coronavirus pneumonia: A review of radiology examination and laboratory test. Journal of Clinical Virology, 128, 104396.

Yates, B., Murphy, D. M., Forrest, I. A., et al. (2005). Azithromycin reverses airflow obstruction in established bronchiolitis obliterans syndrome. Am J Respir Crit Care Med, 172, 772-5. 